\title{
EFFECT OF GRAVITY ON VISCO-ELASTIC SURFACE WAVES IN SOLIDS INVOLVING TIME RATE OF STRAIN AND STRESS OF HIGHER ORDER
}

\author{
TAPAN KUMAR DAS and P. R. SENGUPTA
}

Department of Mathematics, University of Kalyani Kalyani, West Bengal, India

and

\section{LOKENATH DEBNATH}

Department of Mathematics, University of Central Florida

Orlando, Florida 23816, U.S.A.

(Received February 23, 1992 and in revised form March 1, 1993)

\begin{abstract}
A study is made of the surface waves in a higher order visco-elastic solid involving time rate of change of strain and stress under the influence of gravity. A fairly general equation for the wave velocity is derived. This equation is used to examine various kinds of surface waves including Rayleigh waves, Love waves and Stoneley waves. It is shown that the corresponding classical results follow from this analysis in the absence of gravity and viscosity.
\end{abstract}

KEY WORDS AND PHRASES: Surface waves, effects of gravity and viscosity. 1991 AMS SUBJECT CLASSIFICATION CODES: 73D20.

\section{INTRODUCTION}

Considerable literature including Bullen [1], Flugge [2] and Stoneley [3] is available on the theory of surface waves in an isotropic homogeneous elastic solid medium. However, the effects of gravity, viscosity and curvature, although important, are not included in the classical problems. Biot [4] has first investigated the effect of gravity on Rayleigh waves on the surface of an elastic solid based on the assumption that gravity produces a type of initial stress of hydrostatic in nature. Subsequently, Biot's theory has been used by several authors including De and Sengupta $[5,6]$ to study problems of waves and vibrations in solids under the initial stress in various configurations. Further, Sengupta and his associates [7-9] have made an attempt to study the problems of surface waves in solids involving time rate of strain and viscosity. In spite of these studies, relatively less attention has been given to surface wave problems in a higher order visco-elastic solid involving time rate of strain and stress under the influence of gravity. The main purpose of this paper is to study such problems. A fairly general equation for the wave velocity is derived. This equation is utilized to examine various kinds of surface waves including Rayleigh waves, Love waves, and Stoneley waves. It is shown that the corresponding classical results follow from this analysis in the absence of viscosity and gravity.

\section{FORMULATION OF THE PROBLEM AND BOUNDARY CONDITIONS}

Let $M_{1}$ and $M_{2}$ be two homogeneous general visco-elastic solid media involving time rate of strain and stress of higher order in welded contact under the influence of gravity at their common surface of separation. We suppose that the media are separated by a plane horizontal boundary extending to 
infinitely great distance from the origin, $M_{2}$ being above $M_{1}$. We introciuce a set of orthogonal Cartesian coordinate axes $0 x_{1} x_{2} x_{3}$ in the semi-infinite isotropic visco-elastic media, with the origin at the common boundary surface and the $x_{3}$-axis is normal to $M_{1}$. We consider the possibility of a type of wave travelling in the direction of $0 x_{1}$ in such a manner that the disturbance is largely confined to the neighborhood of the boundary and at any instant all particles on any line parallel to $0 x_{2}$ have equal displacements. Hence the wave is a surface wave and all partial derivatives with respect to the coordinate $x_{2}$ are zero. Then the components of displacement $u_{1}$ and $u_{3}$ at any point may be expressed in the form [1]

$$
u_{1}=\frac{\partial \phi}{\partial x_{1}}-\frac{\partial \psi}{\partial x_{3}}, \quad u_{3}=\frac{\partial \phi}{\partial x_{3}}+\frac{\partial \psi}{\partial x_{1}} ;
$$

where $\phi$ and $\psi$ are the functions of $x_{1}, x_{3}$ and $t$ and

$$
\nabla^{2} \phi=\frac{\partial u_{1}}{\partial x_{1}}+\frac{\partial u_{2}}{\partial x_{2}}+\frac{\partial u_{3}}{\partial x_{3}}=\Delta, \quad \nabla^{2} \psi=\frac{\partial u_{3}}{\partial x_{1}}-\frac{\partial u_{1}}{\partial x_{3}} .
$$

Thus the introduction of the functions $\phi$ and $\psi$ enables us to separate out the purely dilational and rotational disturbances associated with the components $u_{1}$ and $u_{3}$. The component $u_{2}$, of course, is associated with purely distortional movement. Thus $\phi, \psi$ and $u_{2}$ are associated respectively with $P$-waves, $S V$-waves and $S H$-waves, as used by Bullen [1].

The stress-strain relations are

$$
D_{\eta} \sigma_{i j}=D_{\lambda} \Delta \delta_{i j}+2 D_{\mu} e_{i j},
$$

where

$$
D_{\eta}=\sum_{k=0}^{n} \eta_{k} \frac{\partial^{k}}{\partial t^{k}}, \quad D_{\lambda}=\sum_{k=0}^{n} \lambda_{k} \frac{\partial^{k}}{\partial t^{k}}, \quad D_{\mu}=\sum_{k=0}^{n} \mu_{k} \frac{\partial^{k}}{\partial t^{k}}
$$

where $\eta_{0}, \lambda_{0}$ and $\mu_{0}$ are the elastic constants and $\eta_{k}, \lambda_{k}$ and $\mu_{k}(k=1,2, \ldots, n)$ are the effects of viscosity, $e_{i j}$ is the strain tensor and $\delta_{i j}$ is the Kronecker symbol.

The displacement equations of motion in the higher order general visco-elastic medium, under the influence of gravity, are

$$
\begin{gathered}
\left(D_{\lambda}+D_{\mu}\right) \frac{\partial \Delta}{\partial x_{1}}+D_{\mu} \nabla^{2} u_{1}+\rho g D_{\eta} \frac{\partial u_{3}}{\partial x_{1}}=\rho D_{\eta} \frac{\partial^{2} u_{1}}{\partial t^{2}}, \\
D_{\mu} \nabla^{2} u_{2}=\rho D_{\eta} \frac{\partial^{2} u_{2}}{\partial t^{2}}, \\
\left(D_{\lambda}+D_{\mu}\right) \frac{\partial \Delta}{\partial x_{3}}+D_{\mu} \nabla^{2} u_{3}-\rho g D_{\eta} \frac{\partial u_{1}}{\partial x_{1}}=\rho D_{\eta} \frac{\partial^{2} u_{3}}{\partial t^{2}},
\end{gathered}
$$

where $\rho, \eta_{k}, \lambda_{k}, \mu_{k}(k=0,1,2, \ldots, n)$ denote the properties of the medium $M_{1}$ and those with dashes the properties of the medium $M_{2}$. Substituting (2.1ab) in equations (2.5)-(2.7), we obtain the wave equations in $M_{1}$ satisfied by $\phi, \psi$ and $u_{2}$, as

$$
\begin{gathered}
\frac{\partial^{2} \phi}{\partial t^{2}}=\mathcal{D}_{T} \nabla^{2} \phi+g \frac{\partial \psi}{\partial x_{1}}, \\
\frac{\partial^{2} \psi}{\partial t^{2}}=\mathcal{D}_{S} \nabla^{2} \psi-g \frac{\partial \phi}{\partial x_{1}}, \\
\frac{\partial^{2} u_{2}}{\partial t^{2}}=\mathcal{D}_{S} \nabla^{2} u_{2},
\end{gathered}
$$

where 


$$
\left.V_{k T}^{2}=\left(\lambda_{k}+2 \mu_{k}\right) / \rho, \quad V_{k S}^{2}-\mu_{k} / \rho\right)
$$

and

$$
\mathcal{D}_{1}=\sum_{k=0}^{n} V_{k T}^{2} \frac{\partial^{k}}{\partial t^{k}} / \sum_{k=0}^{n} \eta_{k} \frac{\partial^{k}}{\partial t^{k}}, \mathcal{D}_{S}=\sum_{k=0}^{n} V_{k S}^{2} \frac{\partial^{k}}{\partial t^{k}} / \sum_{k=0}^{n} \eta_{k} \frac{\partial^{k}}{\partial t^{k}}
$$

and similar relations in $M_{2}$ with $\rho, \eta_{k}, \lambda_{k}, \mu_{k}$ replaced by $\rho^{\prime}, \eta_{k}^{\prime}, \lambda_{k}^{\prime}, \mu_{k}^{\prime}$ and so on (where $k=0,1,2, \ldots, n$ ).

The boundary conditions are

(i) The components of displacement at the boundary surface between the media $M_{1}$ and $M_{2}$ must be continuous at all times and distances.

(ii) The stresses $\sigma_{31}, \sigma_{32}, \sigma_{33}$ are

$$
\begin{aligned}
& D_{\eta} \sigma_{31}=D_{\mu}\left(2 \frac{\partial^{2} \phi}{\partial x_{1} \partial x_{3}}+\frac{\partial^{2} \psi}{\partial x_{1}^{2}}-\frac{\partial^{2} \psi}{\partial x_{3}^{2}}\right), \\
& D_{\eta} \sigma_{32}=D_{\mu} \frac{\partial u_{2}}{\partial x_{3}}, \\
& D_{\eta} \sigma_{33}=D_{\lambda} \nabla^{2} \phi+2 D_{\mu}\left(\frac{\partial^{2} \phi}{\partial x_{3}^{2}}+\frac{\partial^{2} \psi}{\partial x_{1} \partial x_{3}}\right),
\end{aligned}
$$

and similar expressions for $M_{2}$, across the boundary surface between $M_{1}$ and $M_{2}$ must be continuous at all times and distances.

\section{SOLUTION OF THE PROBLEM}

To solve equations (2.8)-(2.10), we put

$$
\left(\phi, \psi, u_{2}\right)=\left[\tilde{\phi}\left(x_{3}\right), \tilde{\psi}\left(x_{3}\right), \bar{u}_{2}\left(x_{3}\right)\right] e^{\iota\left(\eta_{1}-\omega x\right)},
$$

for medium $M_{1}$ and similar solutions for $M_{2}$, the functions $\bar{\phi}, \bar{\psi}, \tilde{u}_{2}$ being replaced by $\tilde{\phi}^{\prime}, \bar{\psi}^{\prime}, \tilde{u}_{2}^{\prime}$.

Introducing (3.1) in (2.8)-(2.10), we have for the medium $M_{1}$ :

$$
\begin{gathered}
{\left[\frac{d^{2}}{d x_{3}^{2}}-\left(\eta^{2}-\omega^{2} \eta_{k}^{*} / V_{k T}^{* 2}\right)\right] \tilde{\phi}=-i g \eta \tilde{\psi} \eta_{k}^{*} / V_{k T}^{* 2},} \\
{\left[\frac{d^{2}}{d x_{3}^{2}}-\left(\eta^{2}-\omega^{2} \eta_{k}^{*} / V_{k S}^{* 2}\right)\right] \tilde{\psi}=i g \eta \tilde{\phi} \eta_{k}^{*} / V_{k S}^{* 2},} \\
{\left[\frac{d^{2}}{d x_{3}^{2}}-\left(\eta^{2}-\omega^{2} \eta_{k}^{*} / V_{k S}^{* 2}\right)\right] \tilde{u}_{2}=0,}
\end{gathered}
$$

where

$$
\dot{\eta_{k}}=\sum_{k=0}^{n}(-i \omega)^{k} \eta_{k}, \quad V_{k T}^{* 2}=\sum_{k=0}^{n}(-i \omega)^{k} V_{k T}^{2}, \quad V_{k s}^{* 2}=\sum_{k=0}^{n}(-i \omega)^{k} V_{k s}^{2}
$$

Similar relations for $M_{2}$ can be obtained by replacing $\tilde{\phi}, \tilde{\psi}, \tilde{u}_{2}, \eta_{k}, V_{k T}, V_{k S}, \eta_{k}^{*}, V_{k T}^{*}, V_{k S}^{*}, \lambda_{k}, \mu_{k}, \rho$ by the same symbols with dashes. Here $\rho^{\prime}, \eta_{k}^{\prime}, \lambda_{k}^{\prime}, \mu_{k}^{\prime}(k=0,1,2, \ldots, n)$ are the physical properties of the medium $M_{2}$.

We assume that $\phi, \psi$ and $u_{3}$ represent exponentially decaying solutions in the medium $M_{1}$ as $x_{3} \rightarrow \infty$ so that they can be expressed in the form:

$$
\phi=\left[A_{1} e^{-x_{3} \sqrt{\eta^{2}-\zeta_{1}^{2}}}+A_{2} e^{-x_{3} \sqrt{\eta^{2}-\xi_{2}^{2}}}\right] e^{u\left(\eta x_{1}-\omega t\right)}
$$




$$
\begin{aligned}
& \psi=\left[B_{1} e^{-x_{3} \sqrt{\eta^{2}-\xi_{1}^{2}}}+B_{2} e^{-x_{3} \sqrt{\eta^{2}-\tau_{2}^{2}}}\right] e^{d\left(\eta x_{1}-\omega t\right)} \\
& u_{2}=\left[C e^{-x_{3} \sqrt{\eta^{2}-\omega^{2} \eta_{k^{\prime}} \cdot v_{k}^{*} \frac{2}{5}}}\right] e^{d\left(\eta x_{1}-\omega t\right)}
\end{aligned}
$$

and similar solutions in $M_{2}$ can be obtained replacing $\phi, \psi, u_{2}, A_{1}, A_{2}, B_{1}, B_{2}, C, \eta_{k}^{*}, V_{k}^{*}, \zeta_{1}, \zeta_{2}$ the same symbols with dashes in solutions (3.6)-(3.8). Here $\zeta^{2}$ and $\zeta_{,}^{\prime}(j-1,2)$ are respectively the roots of the equations

$$
\begin{array}{r}
{\left[\omega^{2}-\zeta^{2} V_{k S}^{* 2} m_{k}^{*}\right]\left[\omega^{2}-\zeta^{2} V_{k T}^{* 2} / \eta_{k}^{*}\right]-g^{2} \eta^{2}=0} \\
{\left[\omega^{2}-\zeta^{\prime 2} V_{k S}^{* \prime 2} / \eta_{k}^{*}\right]\left[\omega^{2}-\zeta^{\prime 2} V_{k T}^{* 2} / \eta_{k}^{*}\right]-g^{2} \eta^{2}=0}
\end{array}
$$

and

$$
B_{1}=\alpha_{1} A_{1}, \quad B_{2}=\alpha_{2} A_{2} ; \quad B_{1}^{\prime}=\alpha_{1}^{\prime} A_{1}^{\prime}, \quad B_{2}^{\prime}=\alpha_{2}^{\prime} A_{2}^{\prime}
$$

where

$$
\alpha_{j}=i g \eta /\left(\omega^{2}-\zeta_{j}^{2} V_{k S}^{* 2} \eta_{k}^{*}\right), \quad \alpha_{j}^{\prime}=i g \eta /\left(\omega^{2}-\zeta_{j}^{\prime 2} V_{k S}^{* \prime 2} m_{k}^{* \prime}\right) \quad(j-1,2)
$$

In evaluating quantities like $\sqrt{\eta^{2}-\zeta^{2}}$ and $\sqrt{\eta^{2}-\omega^{2} \eta_{k}^{*} / V_{k S}^{2}}$, the root with positive real part must be taken in each case.

Using boundary conditions (i) and (ii), we obtain

$$
\begin{aligned}
& {\left[1-i \alpha_{1} Q_{1}\right] A_{1}+\left[1-i \alpha_{2} Q_{2}\right] A_{2}=\left[1+i \alpha_{1}^{\prime} Q_{1}^{\prime}\right] A_{1}^{\prime}+\left[1+i \alpha_{2}^{\prime} Q_{2}^{\prime}\right] A_{2}^{\prime}} \\
& C=C^{\prime} \\
& {\left[\alpha_{1}+i Q_{1}\right] A_{1}+\left[\alpha_{2}+i Q_{2}\right] A_{2}=\left[\alpha_{1}^{\prime}-i Q_{1}^{\prime}\right] A_{1}^{\prime}+\left[\alpha_{2}^{\prime}-i Q_{2}^{\prime}\right] A_{2}^{\prime}} \\
& \rho\left(V_{k S}^{* 2} / m_{k}^{*}\right)\left[\left\{2 i Q_{1}+\left(1+Q_{1}^{2}\right) \alpha_{1}\right\} A_{1}+\left\{2 i Q_{2}+\left(1+Q_{2}^{2}\right) \alpha_{2}\right\} A_{2}\right]= \\
& \rho^{\prime}\left(V_{k S}^{* \prime 2} / \eta_{k}^{\prime \prime}\right)\left[\left\{-2 i Q_{1}^{\prime}+\left(1+Q_{1}^{\prime 2}\right) \alpha_{1}^{\prime}+\left\{-2 i Q_{2}^{\prime}+\left(1+Q_{2}^{\prime 2}\right) \alpha_{2}^{\prime}\right\} A_{2}^{\prime}\right]\right. \\
& -\rho\left(V_{k S}^{* 2} / \eta_{k}^{*}\right) \sqrt{\eta^{2}-\omega^{2} \eta_{k}^{*} / V_{k S}^{* 2}} C=\rho^{\prime}\left(V_{k S}^{* \prime 2} / \eta_{k}^{*}\right) \sqrt{\eta^{2}-\omega^{2} \eta_{k}^{* \prime} / V_{k S}^{* \prime 2}} C^{\prime} \\
& -\left(\rho / \eta_{k}^{* \prime}\right)\left[\left\{V_{k T}^{* \prime 2}\left(Q_{1}^{\prime 2}-1\right)+2 V_{k S}^{* \prime 2}\left(1+i \alpha_{1}^{\prime} Q_{1}^{\prime}\right)\right\} A_{1}^{\prime}+\left\{V_{k T}^{* \prime 2}\left(Q_{2}^{\prime 2}-1\right)+2 V_{k S}^{* \prime 2}\left(1+i \alpha_{2}^{\prime} Q_{2}^{\prime}\right)\right\} A_{2}^{\prime}\right] \text {. }
\end{aligned}
$$

$\left(\rho / \eta_{k}^{*}\right)\left[\left\{V_{k T}^{* 2}\left(Q_{1}^{2}-1\right)+2 V_{k S}^{* 2}\left(1-i \alpha_{1} Q_{1}\right)\right\} A_{1}+\left\{V_{k T}^{* 2}\left(Q_{2}^{2}-1\right)+2 V_{k S}^{* 2}\left(1-i \alpha_{2} Q_{2}\right)\right\} A_{2}\right]$

It follows from equations (3.11b) and (3.11e) that $C=C^{\prime}=0$. Thus there is no propagation of displacement $u_{2}$. Hence there are no $S H$-waves in this case.

From equations (3.11a), (3.11c), (3.11d) and (3.11f), we eliminate the constants $A_{1}, A_{2}, A_{1}^{\prime}, A_{2}^{\prime}$, to get equation for the wave velocity in determinant form

$$
\left|M_{i j}\right|=0, \quad(i, j=1,2,3,4),
$$

where 


$$
\begin{aligned}
& M_{1 m}=\left[1-\imath \alpha_{m} Q_{m}\right], \quad M_{1 m+2}=-\left[1+\imath u_{m} Q_{m}^{\prime}\right] ; \\
& M_{2 m}=\left[\alpha_{m}+i Q_{m}\right], \quad M_{2 m+2}=-\left[\alpha_{m}^{\prime}-i Q_{m}^{\prime}\right] ; \\
& M_{3 m}=\left(\rho V_{k . s}^{\bullet 2} h \eta_{k}^{\cdot}\right)\left[2{ }^{\prime} Q_{m}+\left(1+Q_{m}^{2}\right) \ell_{m}\right] \text {, } \\
& M_{3 m+2}=\left(-\rho V_{k}^{*} \dot{s}_{k}^{\prime}\right)\left[-2 \imath Q_{m}^{\prime}+\left(1+Q_{m}^{\prime 2}\right)\left(x_{m}^{\prime}\right]:\right. \\
& M_{4 m}=\left(\rho / \eta_{k}^{*}\right)\left[V_{k l}^{* 2}\left(Q_{m}^{2}-1\right)+2 V_{k j}^{*}\left(1-i \alpha_{m} Q_{m}\right)\right], \\
& M_{4 m+2}=\left(-\rho^{\prime} / \eta_{k}^{*}\right)\left[V_{k I}^{* \prime 2}\left(Q_{m}^{\prime 2}-1\right)+2 V_{k . S^{*}}^{*}\left(1+i\left(\alpha_{m}^{\prime} Q_{m}^{\prime}\right)\right]\right.
\end{aligned}
$$

and where $m=1,2$.

$$
Q_{m}=\sqrt{1-\zeta_{m}^{2} / \eta^{2}}, \quad Q_{m}^{\prime}-\sqrt{1-\zeta_{m}^{2} / \eta^{2}} .
$$

Equation (3.12) gives the wave velocity for the surface waves in the common boundary, and the strain rate and the stress rate of higher order in the presence of gravity and viscosity are included in (3.12).

\section{PARTICULAR CASES}

(i) Rayleigh Waves

In order to investigate the possibility of Rayleigh waves, we take the plane boundary as a free surface with $M_{2}$ replaced by a vacuum. Obviously, there are no $S H$-waves in this case. In view of (3.11d) and (3.11f), we obtain

$$
\left\{2 i Q_{1}+\left(1+Q_{1}^{2}\right) \alpha_{1}\right\} A_{1}+\left\{2 i Q_{2}+\left(1+Q_{2}^{2}\right) \alpha_{2}\right\} A_{2}=0,
$$

and

$$
\left\{V_{k T}^{* 2}\left(Q_{1}^{2}-1\right)+2 V_{k S}^{* 2}\left(1-i \alpha_{1} Q_{1}\right)\right\} A_{1}+\left\{V_{k T}^{* 2}\left(Q_{2}^{2}-1\right)+2 V_{k s}^{* 2}\left(1-i \alpha_{2} Q_{2}\right)\right\} A_{2}=0 .
$$

Eliminating the constants $A_{1}$ and $A_{2}$ from equations (4.1)-(4.2), we get

$$
\left|M_{i}^{\prime}\right|=0 \quad(i, j=1,2)
$$

where

$$
M_{1 r}^{\prime}=\left[2 i Q_{r}+\left(1+Q_{r}^{2}\right) \alpha_{r}\right] ; \quad M_{2 r}^{\prime}=\left[V_{k T}^{* 2}\left(Q_{r}^{2}-1\right)+2 V_{k S}^{* 2}\left(1-i \alpha_{r} Q_{r}\right)\right] ; \quad(r=1,2) .
$$

Equation (4.3) is the required wave velocity equation for visco-elastic Rayleigh waves including the strain rate and stress rate of higher order under gravitational field. When the effects of viscosity and gravity are neglected, this equation reduces to the classical result as discussed by Bullen [1].

(ii) Love Waves

In this case we consider a layered semi-infinite medium in which $M_{2}$ is bounded by two horizontal plane surfaces at a finite distance $H$-apart, while $M_{1}$ remains infinite as it was. In this case, we consider the displacement component $u_{2}$ only.

For the medium $\boldsymbol{M}_{2}$ we write down the full solution, since the displacement in $\boldsymbol{M}_{2}$ may no longer diminish with the increasing distance from common boundary $x_{3}=0$ and for the medium $M_{1}$ the solutions are the same as it was in the general case.

Therefore, for the medium $M_{2}$ we write

$$
u_{2}^{\prime}=\left[C_{1}^{\prime} e^{x_{3} \sqrt{\eta^{2}-\omega^{2} \eta_{k}^{\prime \prime} N_{k s}^{\prime \prime 2}}}+C_{2}^{\prime} e^{-x_{3} \sqrt{\eta^{2}-\omega^{2} \eta_{k}^{\prime} N_{k s}^{\prime \prime 2}}}\right] e^{t\left(\eta x_{1}-\omega t\right)},
$$


where the restriction that the real part of $\sqrt{\eta^{2}-\omega^{2} \eta_{k}^{*} / V_{k s}^{*}}$ is positive is not required for $M_{\text {? }}$.

In this case the boundary conditions are

(i) $u_{2}$ and $\sigma_{32}$ are continuous at $x_{3}=0$ and (ii) $\sigma_{32}=0$ at $x_{3}=-H$.

Applying these boundary conditions and using (3.8) and (4.5), we find

$$
\begin{aligned}
& C=C_{1}^{\prime}+C_{2}^{\prime} \\
& \left(-\rho V_{k}^{* 2} / \eta_{k}^{*}\right) \sqrt{\eta^{2}-\omega^{2} \eta_{k}^{*} / V_{k S}^{* 2}}=\left[C_{1}^{\prime}-C_{2}^{\prime}\right]\left(\rho^{\prime} V_{k S}^{* 2} / \eta_{k}^{*}\right) \sqrt{\eta^{2}-\omega^{2} \eta_{k}^{*} / V_{k:}^{* \prime 2}}
\end{aligned}
$$

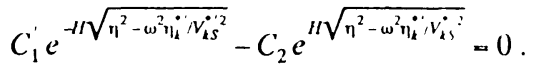

Eliminating $C, C_{1}^{\prime}$ and $C_{2}^{\prime}$ from the equations (4.6)-(4.8), we obtain

$$
\left(\rho V_{k S}^{* 2} / \eta_{k}^{*}\right) \sqrt{1-c^{2} \eta_{k}^{*} / V_{k S}^{* 2}}+\left(\rho^{\prime} V_{k S}^{* 2} / \eta_{k}^{*}\right) \sqrt{\left(c^{2} \eta_{k}^{*} / V_{k S}^{* 2}\right)-1} \tan \eta H \sqrt{\left(c^{2} \eta_{k}^{* /} / V_{k S}^{* 2}\right)-1}-0
$$

where $c=\omega / \eta$. This is the required wave velocity equation for higher order visco-elastic Love waves involving the strain rate and stress rate under the influence of gravity. It is important to note that Iove waves are not affected by gravity but by viscosity. When $\eta_{0}=1$ and $\eta_{k}=\eta_{k}^{\prime}=\lambda_{k}=\lambda_{k}^{\prime}=\mu_{k}=\mu_{k}^{\prime}=0$ $(k=1,2, \ldots, n)$ then equation (4.9) is in agreement with the corresponding classical result $[1]$ in a perfectly elastic medium.

\section{(iii) Stoneley Waves}

In the classical theory, the Stoneley waves are a generalized from of Rayleigh waves propagating along the common boundary of $M_{1}$ and $M_{2}$. In the influence of gravity, Stoneley waves along the common boundary of the general visco-elastic solid media $M_{1}$ and $M_{2}$ involving the strain rate and stress rate of higher order, are therefore determined by the roots of the frequency equation (3.12). In the absence of these effects, this equation also agrees with the corresponding classical result.

\section{REFERENCES}

[1] BULLEN, K. E. An Introduction to the Theory of Seismology, Cambridge University Press, London (1965), p. 253.

[2] FLUGGE, W. Visco-elasticity, Blaisdell Publishing Co. (1967).

[3] STONELEY, R. Proc. R. Soc. London, A. 106 (1924), 416-428.

[4] BIOT, M. A. Mechanics of Incremental Deformation (1965), 44-45, 273-281, Wiley, New York.

[5] DE, S. N. and SENGUPTA, P. R. Ger. Beitr. Geophys., 84 (1975), 509-514.

[6] DE, S. N. and SENGUPTA, P. R. Ger. Beitr. Geophys., 85 (1976), 311-318.

[7] SENGUPTA, P. R. and ROY, S. K. Ger. Beitr. Geophys., 22 (1983), 570-576.

[8] SENGUPTA, P. R. and PAL, K. C. Proc. Ind. Natn. Acad., 53A, No.1 (1987), 113-123.

[9] DAS, T. K. and SENGUPTA, P. R. Ind. J. Pure Appl. Math., 21 (7) (1990), 661-675. 


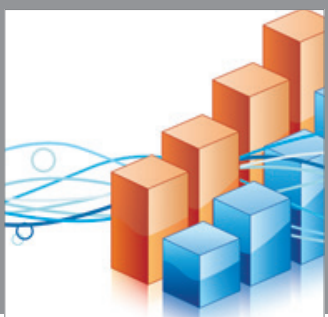

Advances in

Operations Research

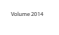

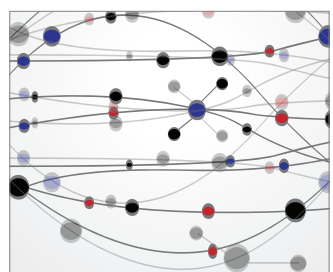

\section{The Scientific} World Journal
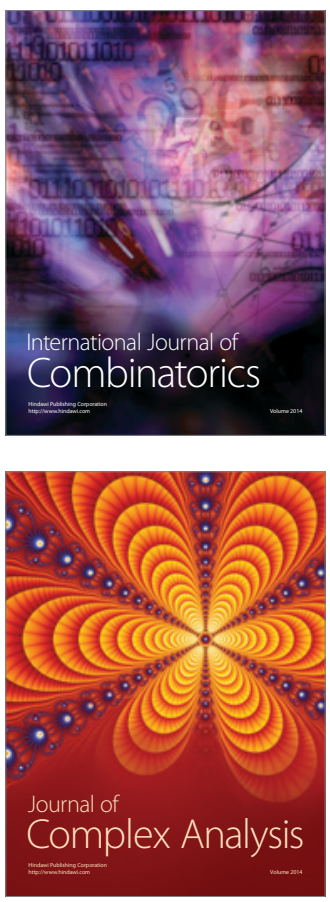

International Journal of

Mathematics and

Mathematical

Sciences
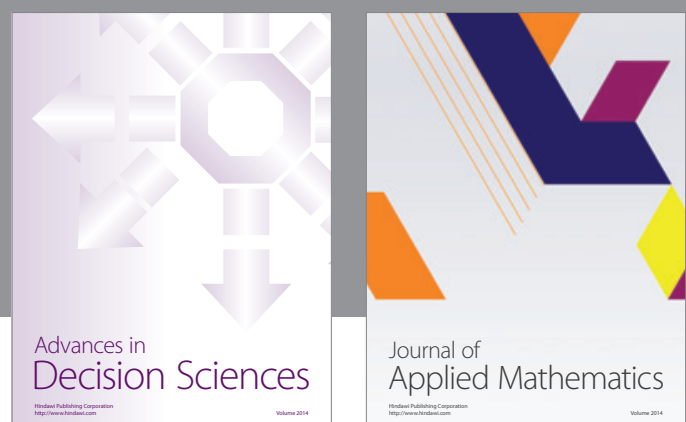

Journal of

Applied Mathematics
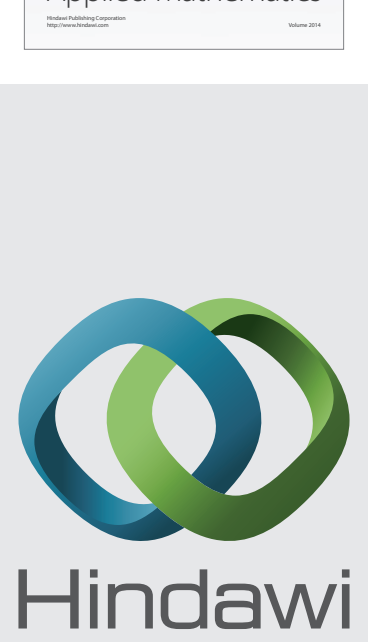

Submit your manuscripts at http://www.hindawi.com
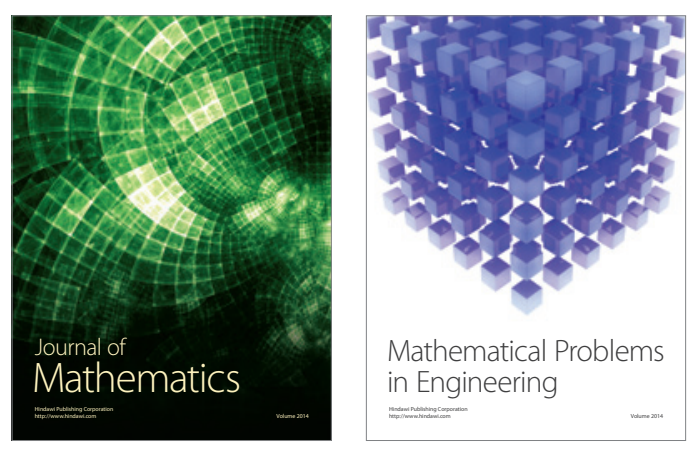

Mathematical Problems in Engineering
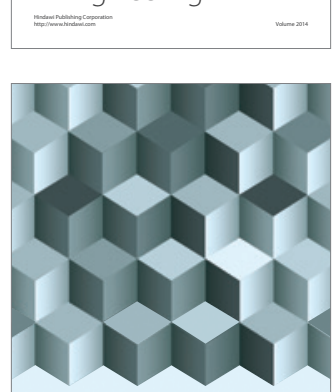

Journal of

Function Spaces
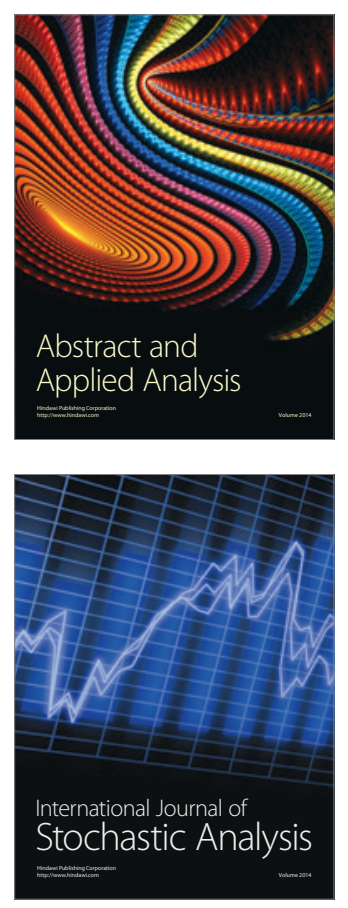

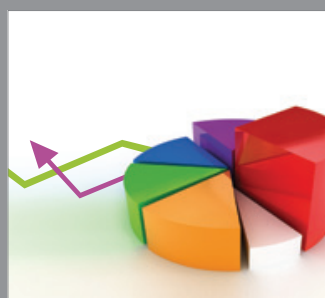

ournal of

Probability and Statistics

Promensencen
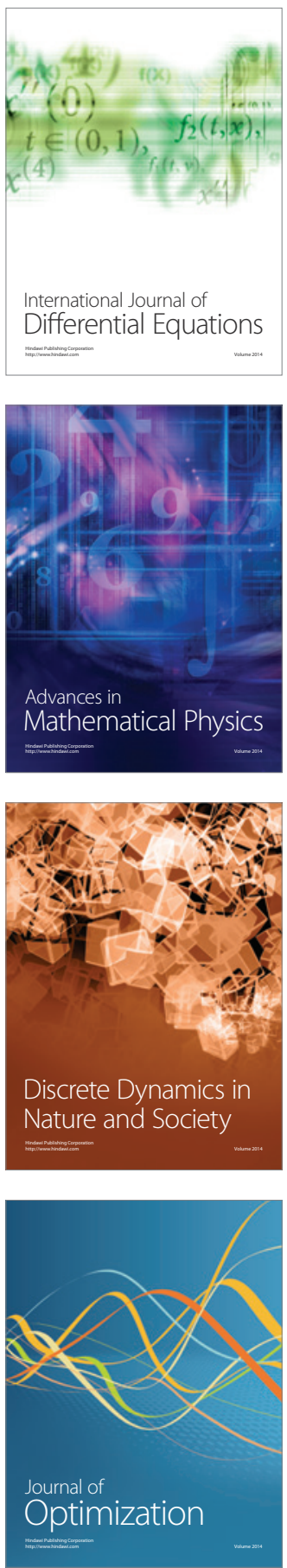